\title{
Efficient electron cooling in Cold Electron Bolometers
}

\author{
$\underline{\text { A.V. Gordeeva }}^{1,2}$, A.L. Pankratov ${ }^{1,2}$, V.O. Zbrozhek $^{1}$, \\ A.V. Blagodatkin ${ }^{1,2}$, L.S. Revin ${ }^{1,2}$, D.A. Pimanov ${ }^{1}$, L.S. Kuzmin ${ }^{1,3}$ \\ ${ }^{1}$ Nizhny Novgorod State Technical University n.a. R.E. Alekseev, Nizhny Novgorod, Russia, anna.gord@list.ru \\ ${ }^{2}$ Institute for Physics of Microstructures of RAS, Nizhny Novgorod, Russia \\ ${ }^{3}$ Chalmers University of Technology, Gothenburg, Sweden
}

We have reached experimentally a theoretical minimum of electron cooling in Cold Electron Bolometer at base temperature $300 \mathrm{mK}$. The efficiency of the cooling depends on how the removal of hot quasiparticles from the vicinity of the tunnel barrier is organized. By double stock and traps for hot quasiparticles we have managed to make the removal of the hot quasiparticles as efficient as $99 \%$. We also explain such high efficiency by the absence of Andreev reflections due to hybrid superconductor/ferromagnet structure for absorber.

The superconductor-insulator-normal metal (SIN) tunnel junctions are known for their ability to remove heat from the electron system of the normal metal electrode. Two SIN junctions, connected in SINIS structure, remove the heat twice more efficiently than a single junction [1]. This property is intensively used in Cold-Electron Bolometers [2,3], where normal metal serves as an absorber of incoming signal.

The maximum cooling by $200 \mathrm{mK}$ was demonstrated in several groups, for example, from $300 \mathrm{mK}$ to $100 \mathrm{mK}$ in [4]. However, the theory predicts that the electron system can be cooled even lower down to $70 \mathrm{mK}$.

The efficiency of the cooling depends on how the removal of hot quasi-particles from the vicinity of the tunnel barrier is organized. In SINIS structures if hot quasi-particles stay near the barrier in S-electrodes, some power may return back to the normal metal through the phonon system or due to backtunneling.

We present three types of cold electron bolometers with different cooling efficiency. We start from the first design, when about $30 \%$ of the heat, removed through the SIN junctions, return back to the absorber. Then we describe the second and the third designs with improvements, that made the return power to decrease from $30 \%$ to $6 \%$ and finally to just $0.5 \%$. Thus, we remove the hot quasiparticles from the vicinity of the tunnel barrier in the most effective possible way.

The tunneling current in SIN junction is given by the well known expression:

$$
I=1 /\left(e R_{T}\right) \int v(\varepsilon)\left[f\left((\varepsilon-e V) / \tau_{E}\right)-f\left(\varepsilon / \tau_{S}\right)\right],
$$

where $V$ - voltage drop across one SIN junction, $R_{T}$ normal state resistance of SIN junction, $v(\varepsilon)$ - density of states in superconductor, $f(\varepsilon)$ - Fermi-Dirac distribution, $\tau_{E}$ and $\tau_{S}$ are electron temperatures in normal metal and in superconductor, respectively. We use the expression (1) to restore electron temperature in the normal absorber together with the heat balance equation, as described, for example, in [5]. The current $I$, voltage $V$ and $R_{T}$ are determined experimentally, $\tau_{S}$ does not influence the result and can be set to the phonon temperature. Thus, only $\tau_{E}$ remains unknown quantity in this expression.

The electron temperatures, found from (1), are shown in Fig. 1 and Fig. 2 on right axis. Normal resistance of the measured samples varies from 0.7 to 1.6 $\mathrm{k} \Omega$. The samples are made of aluminum with critical temperature $1.24 \mathrm{~K}$.

In Fig. 1 we compare a sample with a single connection of superconducting electrode to the thermal bath with a sample, which has two connections to thermal bath for each superconducting electrode. One can see a dramatic increase in electron cooling for the second sample. The better cooling efficiency is seen on IV-curves as increasing superconducting gap. We have measured the critical temperatures of superconducting electrodes for all our samples and found that it was $1.47 \mathrm{~K}$ for the first sample and $1.24 \mathrm{~K}$ for the second one, i.e. it would have opposite effect. Therefore, the change in IV-curves is due to different electron temperatures in the absorber.

We note that the first sample was expected to show good cooling properties as well due to implementation of normal metal traps below the superconducting electrodes. But as appears, the double stock works much more effectively than the single stock with traps.

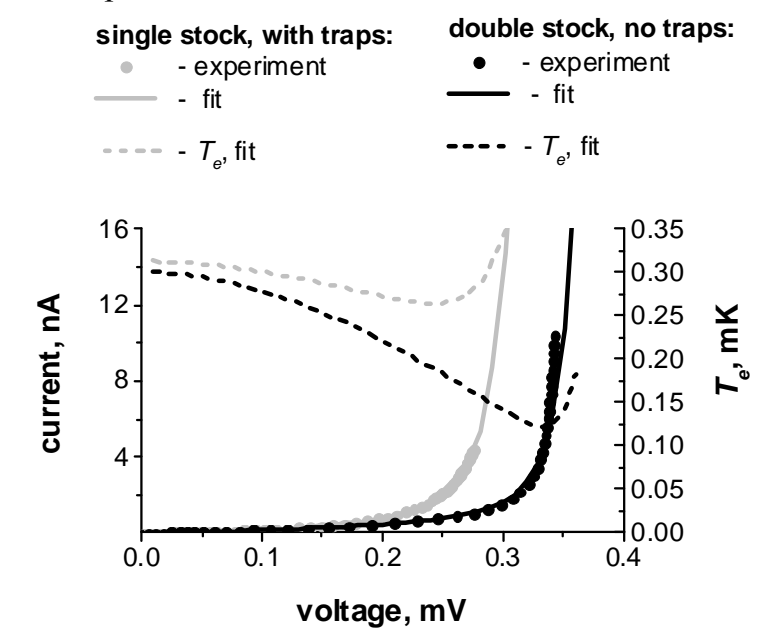

Fig. 1. Left axis: IV-curves of two bolometers with different configuration of superconducting electrodes. Right axis: electron temperatures of the absorbers. The phonon temperature is $300 \mathrm{mK}$ for both bolometers. 
In Fig. 2 we compare the second sample from Fig. 1 with a sample, which has both double stock and traps. Again we can see a significant improvement in cooling properties for the latter sample (black colour).

Now let us point out another possible reason of reaching the minimal electron temperatures, namely suppression of Andreev reflection. It was shown in [6] that two-particle current due to Andreev reflection generates Joule heating in normal electrode, restricting the cooling effect at low temperatures. In our samples we use sublayer of $\mathrm{Fe}$ in order to suppress superconductivity in aluminum, used as a normal metal absorber. We suppose that this thin layer of ferromagnet reduces the Andreev currents in our samples.

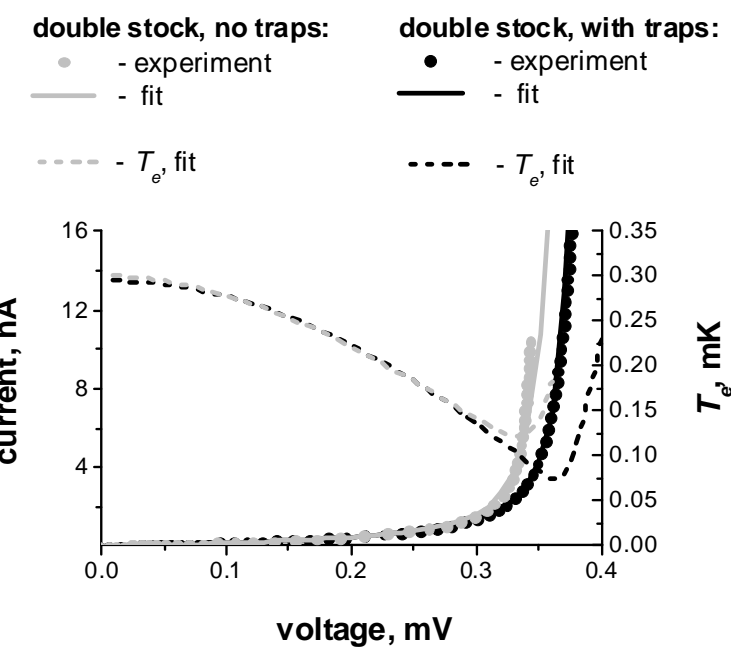

Fig. 2. Left axis: IV-curves of two bolometers without and with normal metal traps below the superconducting electrodes. Right axis: electron temperatures of the absorbers. The phonon temperature is $300 \mathrm{mK}$ for both bolometers.
Thus, by double stock, by traps for hot quasiparticles and by suppression of Andreev current we manage to make the self cooling as efficient as possible. The suppression of Andreev current is organized using hybrid superconductor/ferromagnet structure for the absorber.

Authors would like to thank M. Tarasov and A. Gunbina for help in the sample fabrication. The samples were fabricated in the Chalmers nanotechnology Center. The facilities of the Center of Cryogenic Nanoelectronics of NNSTU and Common Research Center "Physics and technology of micro- and nanostructures" of IPM RAS were used.

The work is supported by Russian Science Foundation (Project 16-19-10468).

\section{References}

1. Nahum M., Eiles T. M. and Martinis J. M. Electronic micro-refrigerator based on a NIS tunnel junction // Appl. Phys. Lett. 1994. V. 65. P. 3123.

2. Kuzmin L. Ultimate Cold-Electron Bolometer with Strong Electrothermal Feedback // Proceedings of SPIE. 2004. V. 5498. P. 349.

3. Kuzmin L. An Array of Cold-Electron Bolometers with SIN Tunnel Junctions and JFET readout for Cosmology Instruments // Journal of Physics: Conference Series. 2008. V. 97, P. 012310.

4. Leivo, M.M., Pekola, J.P. and Averin, D.V. Microrefrigeration by NIS tunnel junctions // Czech J Phys. 1996. V. 46(Suppl 5): P. 2763.

5. Gordeeva,A.V., et al. Observation of photon noise by cold-electron bolometers // Appl. Phys. Lett. 2017. V. 110. P. 162603

6. Vasenko A.S., Bezuglyi E.V., Courtois H., Hekking F.W.J. Electron cooling by diffusive normal metalsuperconductor tunnel junctions // Phys. Rev. B. 2010. V. 81. P. 094513. 